\title{
Management of Breast Cancer Risk in Women with Ovarian Cancer and Deleterious BRCA1 or BRCA2 Mutations
}

\author{
Andrea E. Wahner Hendrickson, $\mathrm{MD}^{1}$, Jamie N. Bakkum-Gamez, $\mathrm{MD}^{2}$, Fergus J. Couch, $\mathrm{PhD}^{3}$, Karthik Ghosh, \\ $\mathrm{MD}^{4}$, and Judy C. Boughey, $\mathrm{MD}^{5}$ \\ ${ }^{1}$ Department of Medical Oncology, Mayo Clinic Rochester, Rochester, MN; ${ }^{2}$ Department of Obstetrics \& Gynecology, \\ Mayo Clinic Rochester, Rochester, MN; ${ }^{3}$ Department of Laboratory Medicine and Pathology, Mayo Clinic Rochester, \\ Rochester, MN; ${ }^{4}$ Department of Medicine, Mayo Clinic Rochester, Rochester, MN; ${ }^{5}$ Department of Surgery, Mayo Clinic \\ Rochester, Rochester, MN
}

Advances in genetic testing have led to a new clinical dilemma: the optimal management of the elevated risk of breast cancer in women with ovarian cancer found to have a deleterious mutation in BRCA1 or BRCA2. In October 2014, the Society of Gynecologic Oncology released a clinical practice statement recommending that all women diagnosed with epithelial ovarian/fallopian tube/peritoneal cancer should receive genetic counseling and be offered genetic testing for genes associated with hereditary breast and ovarian cancer syndrome, even in the absence of family history. The need for the statement was based on the knowledge that approximately $15 \%$ of ovarian cancer patients, irrespective of family history, have germline BRCA1 or BRCA2 mutations. ${ }^{1}$ The National Comprehensive Cancer Network has the same recommendation. ${ }^{2}$ Since the release of these guidelines, an increasing number of women with ovarian cancer are being referred for genetic counseling. Most women referred for genetic counseling complete the consultation and $>90 \%$ opt for genetic testing. ${ }^{3,4}$ Given the clinical availability of PARP inhibitors for treatment of ovarian cancer and the importance of cascade testing in identifying family members eligible for cancer risk reduction strategies, efforts to improve rates of genetic counseling referral are needed. Early findings after implementing a bundled approach to enhance referral indicate that nearly $75 \%$ of women with a new ovarian cancer at

(C) Society of Surgical Oncology 2017

First Received: 2 June 2017;

Published Online: 20 July 2017

A. E. Wahner Hendrickson, MD

e-mail: wahnerhendrickson.andrea@mayo.edu
Mayo Clinic Rochester are now referred for genetic counseling with a goal of universal referral. ${ }^{5}$

Women with BRCA1 and BRCA2 mutations have a $65 \%$ (95\% confidence interval [CI] 51-75\%) and a $45 \%(95 \%$ CI $22-51 \%$ ) chance of developing breast cancer by age 70 years, respectively. ${ }^{6}$ However, because ovarian cancer is the most lethal of gynecologic malignancies, with an overall 5-year survival rate of $45.6 \%$, it is unclear how to manage these patients with an increased risk of breast cancer who have been diagnosed with invasive epithelial ovarian carcinoma.

In the article entitled "Cost effectiveness of risk-reducing mastectomy versus surveillance in BRCA mutation carriers with a history of ovarian cancer," Gamble et al. have approached this question by using modeling studies to determine the survival benefit and cost effectiveness of bilateral risk-reducing mastectomy (RRM) compared with surveillance (annual mammogram and breast MRI) in women with stage II-IV ovarian cancer who were subsequently found to be $B R C A 1$ and $B R C A 2$ mutation carriers. This study is the first to evaluate these options in this specific subset of women and is the first to assess impact in terms of survival as well as cost. Among women older than age 60 years who are diagnosed with ovarian cancer, the incremental cost-effectiveness ratio (ICER) exceeds a generally accepted willingness to pay threshold of $\$ 100,000-\$ 200,000$ per year of life saved (YLS) in most scenarios and the benefit of RRM was negligible. The added benefit of RRM in terms of survival gain in months was greatest in women aged 40 years and was 5 months for BRCA1 carriers and 3.6 months in BRCA2 carriers. At 60 years or older, the survival gain was 1.3 months or less and exceeded ICER thresholds. In women older than age 
TABLE 1 Management recommendations for women with ovarian cancer without evidence of disease and $B R C A 1$ or $B R C A 2$ deleterious mutation

\begin{tabular}{llll}
\hline $\begin{array}{l}\text { Stage at diagnosis of ovarian } \\
\text { cancer }\end{array}$ & $\begin{array}{l}\text { Age at diagnosis of ovarian } \\
\text { cancer }\end{array}$ & RRM & Breast cancer screening $^{\mathrm{a}}$ \\
\hline I & Any & $\begin{array}{l}\text { RRM can be considered }>1 \text { year after } \\
\text { diagnosis } \\
\text { RRM may be considered }>5 \text { years after } \\
\text { diagnosis }\end{array}$ & Recommended \\
II-IIIB & $\leq 50$ & RRM not recommended & May be considered \\
IIIC-IV & $\geq 51$ & RRM not recommended & $\begin{array}{c}\text { Not generally recommended but } \\
\text { individualize }\end{array}$
\end{tabular}

a Annual screening decision to be individualized based on benefits, risks and limitation of the screening tests and accounting for the patient's estimated life-expectancy and ongoing treatments for ovarian cancer

70 years, the survival gain from RRM was less than 1 month.

The study findings suggest that RRM is not indicated within 5 years of ovarian cancer diagnosis in women with stage II-IV disease, with breast surveillance recommended during this time period. This is likely due to the high risk of early relapse of ovarian cancer. However, surgical riskreducing strategies may be considered for patients who are at least 5 years out from diagnosis without ovarian cancer recurrence or progression.

While this study highlights important considerations when discussing risk-reducing efforts for this population, it is important to address certain limitations of this modelling study. The analysis combined stage II to IV disease together and excluded stage I disease. However, the survival rates among women with ovarian cancer differ significantly by stage. Stage I ovarian cancer patients have a 5-year overall survival of approximately $85 \%$ compared with $70 \%$ for stage IIA, $32 \%$ for stage IIIC, and $18.6 \%$ for women with stage IV disease. ${ }^{7}$ Therefore, any discussion of the benefits of RRM in a stage III or IV patient should be cautioned as it is much less applicable than in a patient with stage I or II disease.

This study did not evaluate the possible role of risk-reducing medications (tamoxifen, raloxifene, or aromatase inhibitors) in these patients at high risk for breast cancer. Tamoxifen reduces the risk of contralateral breast cancer in breast cancer patients with $B R C A 1$ or $B R C A 2$ mutations. ${ }^{8,9}$ However, whether tamoxifen is effective in reducing recurrence in $B R C A 1 / 2$ mutation carriers with hormone receptornegative breast cancer is unclear and chemoprevention has not been studied in BRCA1/2 mutation carriers with ovarian cancer. Furthermore, the additive value of these medications in women who have undergone a bilateral salpingooophorectomy (BSO) is not well established. ${ }^{10}$

The findings from this study highlight the importance of length of disease-free interval in the decision making process. Although many ovarian cancer patients do live an extended period of time after diagnosis, these years often are complicated by recurrences with multiple chemotherapy regimens and very short intervals of time without treatment. Adding major surgical interventions (with or without reconstruction) to reduce the risk of breast cancer during chemotherapy-free intervals also has the potential to negatively impact the overall quality of life. Furthermore, patients with early recurrence and platinum-resistant disease are not likely to be ideal candidates for RRM due to the competing risk of death from their ovarian cancer.

As more women with ovarian cancer undergo genetic testing and are found to have $B R C A 1$ or $B R C A 2$ mutations, we must be aware of how best to address their increased risk of breast cancer. At Mayo Clinic, the multidisciplinary team, including gynecologic medical oncologists, gynecologic surgeons, medical geneticists, breast surgeons, and internal medicine breast clinic physicians developed management guidelines for our practice, and we have further revised them to incorporate the data from this article. Our general guideline for practicing clinicians is summarized in Table 1. It is important to take age and stage into consideration when discussing RRM versus surveillance with each patient. In women with stage I ovarian cancer who are disease-free for at least 1 year, it may be very reasonable to discuss RRM, because this is the cohort of patients who are most likely to benefit from this procedure. Because the survival benefit is less than 3 months for women over age 50 years at diagnosis, in most cases we recommend that RRM not be performed in women aged 50 years or older. In the remainder, it should not be considered until patients are disease-free for 5 years, and then stage and age at diagnosis need to be considered. For those patients without ovarian cancer relapse at 5 years, RRM may be considered in patients with stage II-IIIB diagnosed at age $\leq 50$ years. In older women and those with higher stage disease, RRM is not recommended.

In general, in women who are undergoing first-line treatment for their ovarian cancer, it is preferable to wait 
until they have completed therapy and are at least 1 year after diagnosis before referring them to a high-risk Breast Clinic or Breast Surgeon for further evaluation. At that point, a discussion can be held between the physician and the patient with the understanding that the cost-effectiveness of RRM does not appear to reach cost thresholds with minimal impact on survival until these women are 5 years postdiagnosis and is limited to those women younger than age 50 years at the time of ovarian cancer diagnosis.

We must note that this study was not designed to address the role of breast cancer screening. While studies have questioned the role of screening for breast cancer in this population, suffice to state that the decision on the role of screening and the modality to use for screening should be made at an individual level. The benefits of early diagnosis of breast cancer need to be balanced with the potential risks/limitations of false-positive test results, overdiagnosis, additional testing, and anxiety for the patient. Also, decisions need to incorporate the patient's life expectancy from the ovarian cancer itself. For women with recurrent ovarian cancer and advanced-stage disease with short disease-free intervals, the benefit of surveillance has not been evaluated and surveillance in these women may not be indicated.

Gamble et al. have published the first study that helps us to evaluate the survival benefit and cost-effectiveness of RRM among women with BRCA1 and BRCA2 mutations following stage II-IV ovarian cancer. In an era where utilizing our healthcare dollars effectively becomes critical, we applaud the work of this study team for addressing this difficult issue and bringing to light the relatively small amount of survival gained for this invasive procedure in women with ovarian cancer.

\section{REFERENCES}

1. George A, Kaye S, Banerjee S. Delivering widespread BRCA testing and PARP inhibition to patients with ovarian cancer. Nat Rev Clin Oncol. 2017;14(5):284-96.

2. National Comprehensive Cancer Network (NCCN). BRCA-related breast and/or ovarian cancer syndrome. 2017. https://www. nccn.org/professionals/physician_gls/pdf/genetics_screening.pdf.

3. Febbraro T, Robison K, Wilbur JS, et al. Adherence patterns to National Comprehensive Cancer Network (NCCN) guidelines for referral to cancer genetic professionals. Gynecol Oncol. 2015;138(1):109-14.

4. Ricci MT, Sciallero S, Mammoliti S, Gismondi V, Franiuk M, Bruzzi P, et al. Referral of ovarian cancer patients for genetic counselling by oncologists: need for improvement. Public Health Genomics. 2015;18(4):225-32.

5. Swanson $\mathrm{C}$, et al. Increasing genetic counseling referral rates after ovarian cancer diagnosis: improving the quality of ovarian cancer care. Gynecol Oncol. 2016;141(Suppl 1):194.

6. Antoniou A, Pharoah PD, Narod S, et al. Average risks of breast and ovarian cancer associated with BRCA1 or BRCA2 mutations detected in case series unselected for family history: a combined analysis of 22 studies. Am J Hum Genet. 2003;72(5):1117-30.

7. Heintz AP, Odicino F, Maisonneuve P, et al. Carcinoma of the ovary. FIGO 26th annual report on the results of treatment in gynecological cancer. Int J Gynaecol Obstet. 2006;95(Suppl 1):S161-92.

8. Gronwald J, Tung N, Foulkes WD, et al. Tamoxifen and contralateral breast cancer in BRCA1 and BRCA2 carriers: an update. Int $J$ Cancer. 2006;118(9):2281-4.

9. Phillips KA, Milne RL, Rookus MA, et al. Tamoxifen and risk of contralateral breast cancer for BRCA1 and BRCA2 mutation carriers. J Clin Oncol. 2013;31(25):3091-9.

10. Peshkin BN, Isaacs C, Finch C, Kent S, Schwartz MD. Tamoxifen as chemoprevention in BRCA1 and BRCA2 mutation carriers with breast cancer: a pilot survey of physicians. J Clin Oncol. 2003;21(23):4322-8. 